\title{
Optical Frequency Combs for Low Phase Noise Microwave Generation
}

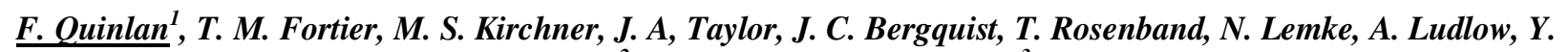 \\ Jiang $^{2}$, C. W. Oates, S. A. Diddams ${ }^{3}$ \\ National Institute of Standards and Technology, 325 Broadway, Boulder CO 80305, Ph. 303-497-3295, Fax: 303- \\ 497-6461 \\ ${ }^{2}$ Current address: East China Normal University, Shanghai, 200062, China \\ ${ }^{1}$ fquinlan@boulder.nist.gov, ${ }^{3}$ sdiddams@boulder.nist.gov
}

\begin{abstract}
An optical frequency comb locked to a stable optical reference can serve as a source for microwave signals having very low close-to-carrier phase noise. This has recently been confirmed by comparing two independent systems, yielding an absolute phase noise of $-104 \mathrm{dBc} / \mathrm{Hz}$ at $1 \mathrm{~Hz}$ offset from a $10 \mathrm{GHz}$ carrier. The corresponding timing jitter is 760 attoseconds, integrated from $1 \mathrm{~Hz}$ to $1 \mathrm{MHz}$. Here we describe the system architecture, as well as technical and fundamental noise limitations.
\end{abstract}

\section{Introduction}

Combining a femtosecond laser optical frequency comb (OFC) with the high quality factor (Q) available in optical cavities allows for the possibility of microwave signals with phase noise below state-of-the-art electronic oscillators. The low loss of optical Fabry-Perot (FP) resonators leads to Q values exceeding $10^{11}$, greater than microwave dielectric resonator oscillators at either room $\left(\mathrm{Q} \sim 10^{5}\right)$ or cryogenic temperatures $\left(\mathrm{Q} \sim 10^{9}\right)$. When an optical frequency comb is phase-locked to an optical reference, the comb can be thought of as a high fidelity frequency divider, transferring the stability of the optical reference to the microwave domain [1-3]. The division in frequency leads to a division in phase noise, where the phase noise power spectral density scales as $S_{\mu-w a v e}^{\phi}=$ $S_{o p t}^{\phi} / N^{2}$. Here, $S^{\phi}$ is the phase noise and $N$ is the optical-to-microwave division ratio. Optical references with a fractional frequency stability of $<3 \cdot 10^{-16}$ (at 1second) have been demonstrated [4], ideally generating a $10 \mathrm{GHz}$ signal with phase noise of $<-110 f^{-3} \mathrm{dBc} / \mathrm{Hz}$. At $1 \mathrm{~Hz}$ offset from the carrier, this represents a $40 \mathrm{~dB}$ improvement over the best room temperature $10 \mathrm{GHz}$ electronic microwave oscillators [5], and is below what has been demonstrated with cryogenic $10 \mathrm{GHz}$ oscillators $[6,7]$. Work with Er:fiber-based frequency combs has shown a residual phase noise in optical-to-microwave conversion of $-118 \mathrm{dBc} / \mathrm{Hz}$ at $1 \mathrm{~Hz}$ offset from a $11.55 \mathrm{GHz}$ carrier [2]. Recently, we demonstrated absolute phase noise below $-104 \mathrm{dBc} / \mathrm{Hz}$ at $1 \mathrm{~Hz}$ offset from a $10 \mathrm{GHz}$ carrier by comparing two independent systems that employ $1 \mathrm{GHz}$ mode-locked Ti:sapphire laser OFCs [3]. This represents one of the lowest close-to-carrier phase noise measurements yet reported on a $10 \mathrm{GHz}$ source. The ability to optically synthesize pure tones or even waveforms with low phase noise in the microwave, millimeter-wave or even terahertz domains benefits a number of scientific and signal processing applications, including high speed analog-todigital conversion, remote synchronization, local oscillators for fountain clocks, coherent control over quantum mechanical processes, and photonically enabled generation and processing of ultrabroadband radiofrequency electrical signals.

\section{Optical Frequency Comb as Optical Frequency Divider}

The OFC in time and frequency domains is shown in Fig. 1(a), and a schematic of the optical frequency divider architecture is shown in Fig. 1(b). For low noise microwave generation, an optical mode of the OFC is phase-locked to a laser that is stabilized to the FP cavity. Pulse formation via passive mode-locking enforces a constant phase among the laser modes. As a result, stabilizing one mode to the optical reference will transfer its stability to every optical mode of the comb [8]. The equation describing the frequency of the comb lines then becomes 


$$
v_{\text {opt }}=n \cdot f_{\text {rep }}+f_{o}+f_{b}
$$

where $v_{\text {opt }}$ is the frequency of the optical reference, $f_{\text {rep }}$ is the combline spacing (or pulse repetition rate), $f_{o}$ is an offset frequency common to all the modes of the $\mathrm{OFC}$, and $f_{b}$ is the difference or beat frequency between the optical reference and a comb mode. We may therefore write $f_{\text {rep }}$ as

$$
f_{\text {rep }}=\left(v_{\text {opt }}-f_{o}-f_{b}\right) / n
$$

Thus $f_{\text {rep }}$ represents the frequency-divided optical reference (most directly seen in the special case of $f_{o}$ and $f_{b}$ equal to zero). Accessing $f_{\text {rep }}$ is achieved by photodetecting the optical pulse train to generate a corresponding electrical pulse train. In the frequency domain, this train of electrical pulses is a series of discrete tones at harmonics of the pulse repetition rate. Any harmonic of $f_{\text {rep }}$ within the photodetector bandwidth can then be selected as a low noise microwave source.

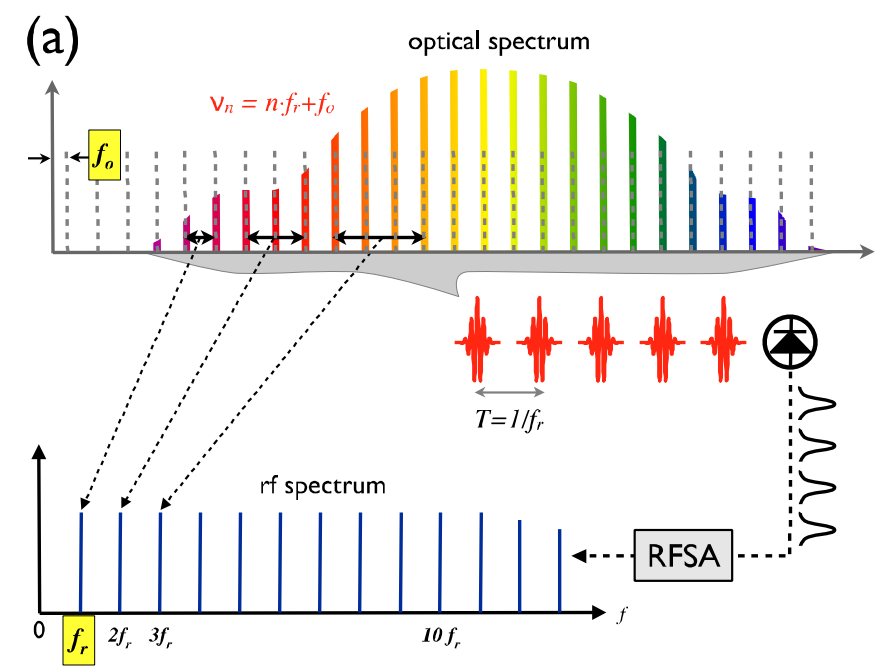

(b)

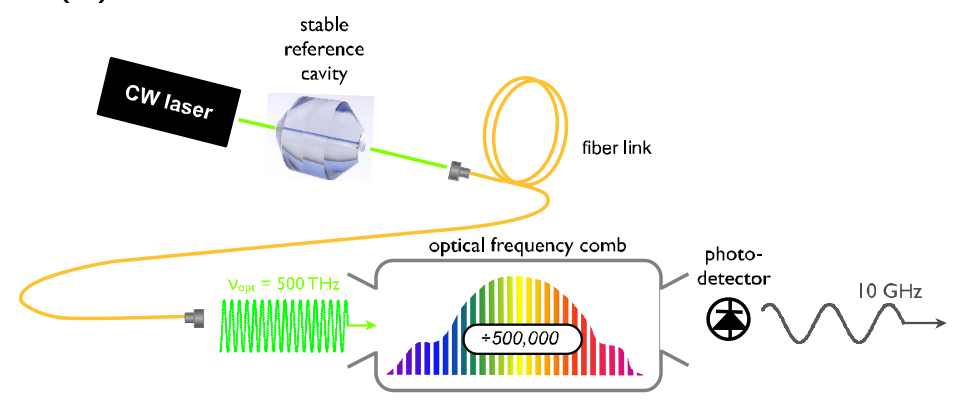

Figure 1 (a) The optical frequency comb in time and frequency domains. Photodetection of the optical pulse train results in a train of electrical pulses. The electrical pulse spectrum is an array of discrete tones at harmonics of the pulse repetition rate. (b) Architecture for microwave generation from an optical reference. When locked to an optical reference, the OFC acts as a high fidelity frequency divider.

\section{Low Phase Noise Microwaves}

Although we may consider the microwave signal as the frequency-divided optical reference, the noise of the resulting microwave signal is not from the optical reference alone. Also contributing are residual noise on the phase lock between the comb and the optical reference, phase noise on the comb offset frequency, phase noise added by the link between the OFC and photodetector, and phase noise added during photodetection. As shown in Fig. 2, from low offset frequency to high offset frequency, the noise is in turn dominated by the optical reference, an unstabilized 
fiber link, the OFC, and photodetection noise. For comparison, the phase noise, scaled to a $10 \mathrm{GHz}$ carrier, of an optical reference at $518 \mathrm{THz}$ is also shown in Fig. 2. This would be the microwave phase noise level if it were determined only by the optical reference.

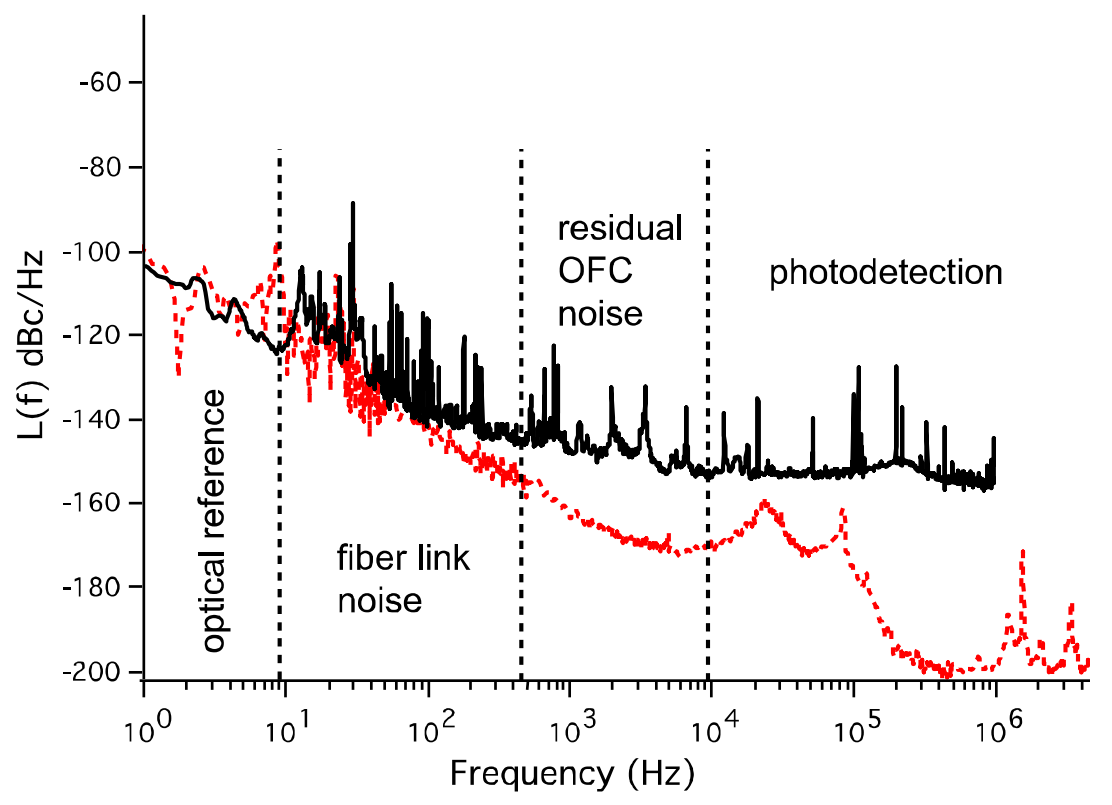

Fig. 2 Phase noise on an optically derived $10 \mathrm{GHz}$ signal and contributing noise sources. Black solid line: measured absolute phase noise at $10 \mathrm{GHz}$. The corresponding timing jitter is 760 attoseconds ( $1 \mathrm{~Hz}-1 \mathrm{MHz}$ integration). Red dotted curve: phase noise of an optical reference, scaled to a $10 \mathrm{GHz}$ carrier.

\section{References}

[1] J. J. McFerran, et al., "Low noise synthesis of microwave signals from an optical source," IEEE Electron. Lett., vol. 41, 2005.

[2] W. Zhang, et al., "Sub-100 attoseconds stability optics-to-microwave synchronization," Applied Physics Letters, vol. 96, May 2010.

[3] T. M. Fortier, et al., "Photonic Generation of Ultrastable $10 \mathrm{GHz}$ Microwave Signals," submitted for publication, 2011.

[4] Y. Y. Jiang, et al., "Making optical atomic clocks more stable with 10-16-level laser stabilization," Nat Photon, vol. 5, pp. 158-161, 2011.

[5] E. N. Ivanov and M. E. Tobar, "Low Phase-Noise Sapphire Crystal Microwave Oscillators: Current Status," IEEE Transactions on Ultrasonics Ferroelectrics and Frequency Control, vol. 56, pp. 263-269, Feb 2009.

[6] S. Grop, P. Y. Bourgeois, R. Boudot, Y. Kersale, E. Rubiola, and V. Giordano, "10 GHz cryocooled sapphire oscillator with extremely low phase noise," Electronics Letters, vol. 46, pp. 420-422, Mar 2010.

[7] A. G. Mann, C. Sheng, and A. N. Luiten, "Cryogenic sapphire oscillator with exceptionally high frequency stability," IEEE Transactions on Instrumentation and Measurement, vol. 50, pp. 519-521, Apr 2001.

[8] A. Bartels, C. W. Oates, L. Hollberg, and S. A. Diddams, "Stabilization of femtosecond laser frequency combs with subhertz residual linewidths," Optics Letters, vol. 29, pp. 1081-1083, May 2004. 